\title{
Laboratory Model Test to Explore the Bearing Mechanism of Composite Foundation in the Loess Area
}

\author{
Longfei Xu $\mathbb{D}^{1},{ }^{1}$ Weijiang Xu ${ }^{\mathbb{D}},{ }^{1}$ Aijun Zhang, ${ }^{2}$ Xiaolin Weng, ${ }^{1}$ Gaowen Zhao, ${ }^{1}$ \\ and Tantan $\mathrm{Zhu}^{1}$ \\ ${ }^{1}$ School of Highway, Chang'an University, Xi'an 710064, China \\ ${ }^{2}$ College of Water Resources and Architectural Engineering, Northwest A\&F University, Yangling, Shaanxi 712100, China
}

Correspondence should be addressed to Weijiang Xu; 2019221094@chd.edu.cn

Received 2 December 2020; Revised 15 February 2021; Accepted 8 March 2021; Published 18 March 2021

Academic Editor: Chunshun Zhang

Copyright (c) 2021 Longfei Xu et al. This is an open access article distributed under the Creative Commons Attribution License, which permits unrestricted use, distribution, and reproduction in any medium, provided the original work is properly cited.

\begin{abstract}
Pile composite foundation can make good use of the bearing capacity of the soil and pile, which is widely used in the Chinese northwest loess area. However, the theory of pile composite foundation is far from sufficient, hindering its long-term development. Aiming at this problem, a laboratory model test of pile composite foundation in the loess area was conducted to explore the common working mechanism and variations of each bearing stage. Besides, the settlement of the single pile composite foundation was calculated by using the modified tangent modulus method, and the result was compared with the experimental data. The main results of this paper are as follows: Both in the single pile and single pile composite foundation, loading-settlement curves showed a trend of "elastic to elastoplastic to plastic," accompanied by the appearance of plummeting point. Influenced by the pile group effect, the loading-settlement curve of the group pile composite foundation showed a slow-varying trend without an obvious breakdown point. Pile axis stress increased with the growth of upper load. At the beginning of loading, the pile axis stress indicated such a distribution that stress on both ends of the pile was larger than that in the middle of the pile. When reaching a certain load, the location of the biggest pile axis stress transferred to the pile top, and the pile axis stress decreased gradually as the pile became deep. The side friction resistance in the static load test of the single pile was always positive, whereas in the composite foundation of a single pile and a group of piles, negative side resistance appeared in the upper side of the neutral point. Pile-soil stress ratio in the depth of $12 \mathrm{~cm}$ changed with the upper load. The outcome calculated by the modified tangent modulus method had a relatively better consistency with experimental data if the upper load was not too large.
\end{abstract}

\section{Introduction}

Loess is widely distributed in northwest China. The foundation plays an important role in the building stability of the loess area. Once the foundation suffers destruction, the safety of the entire building will be endangered and hard to remedy [1]. In recent years, the pile composite foundation has been widely used in construction engineering, transportation engineering, and municipal engineering in the loess area of China due to its advantages. More especially, in the pile composite foundation, the pile and soil can bear the upper load together, which can give full play to the bearing capacity of soil in the foundation. Besides, the construction cost can be drastically reduced. In addition, loess has obvious collapsibility in northwest China, and the pile composite foundation (especially the composite foundation with granular piles) can not only effectively reduce the foundation settlement but also do help to eliminate the collapse of loess [2-4].

At present, the theoretical research of the pile composite foundation is not abundant enough, which is not beneficial to spread the quantitative design method in the engineering field and unfavorable to its development. Moreover, due to the deterioration characteristics of loess (e.g., collapsibility), which makes the bearing mechanism of the pile composite foundation more complex and changeable in the loess area. Aiming at the aforementioned problem, some scholars have carried out plenty of research, which has exerted its profound influence on the development of the pile compound foundation in the loess area of China. As early as 1996, Liu 
et al. [5] combined with the projects in the west and east of Gansu Province and Guanzhong region of Shanxi Province to make a preliminary discussion on the lime soil compaction pile composite foundation and calculation method of the bearing mechanism. Based on the field test, Nie et al. [6-8] discussed the reasonable value of the pile spacing by studying the rammed extrusion piling in the collapsible loess foundation. Moreover, numerical calculation methods are also widely used in the study of the composite pile foundation bearing mechanism [9-15]. Through the finite element method, Feng et al. [9] analyzed the composite foundation formed by the cement injection pile to treat the saturated loess under the embankment flexible foundation, thereby exploring the bearing mechanism and proposing the corresponding bearing capacity calculation formula. Yang et al. [10] explored the stress and deformation mechanism of the PCP (Prestressed Concrete Pipe) pile composite foundation through a field test and finite element model; Soomro et al. [11-13] conducted a numerical parametric study of the settlement and load transfer mechanism of the pile group due to twin-stacked tunneling with different construction sequences.

Nevertheless, the abovementioned analysis is limited by the following aspects: firstly, engineering examples or field tests are often restricted by site conditions, there are a few controllable factors, and it is not easy to repeat. Secondly, due to the complexity of the pile-soil interaction mechanism in the composite foundation, it is difficult to obtain ideal results by a numerical method considering the difficulty of determining reasonable calculation parameters. Recently, the laboratory model test has becoming one of the important means in the theoretical studying of the composite foundation. With this method, the main parameters of the test object can be strictly controlled, and it is not restricted by external or natural conditions. The experimental results are accurate and can be used to verify the conclusions of the prototype. Wang et al. [16] studied the settlement effect of a rigid pile net composite foundation by a centrifugal model test; Zheng et al. [17] studied with loadeffect of a multicolumn composite foundation and proposed the formula for calculating the multicolumn composite foundation; moreover, $\mathrm{Yi}$ et al. [18] conducted experimental research on the carrying capacity, stress distribution, and failure mode of composite foundations through laboratory model testing; based on the analysis of the stability parameters of the composite foundation in the loess area, Cheng et al. [19] concluded that the increase of friction angle or foundation cohesion can significantly improve the safety factor of the composite foundation. Sun et al. [20] studied the bearing capacity of the cement soil pile composite foundation in the collapsible loess area through a field test, which provided reliable test data for future research. In addition, Soomro et al. [21, 22] investigated the effects of different construction sequences on the settlement and load transfer mechanism of the single pile due to twin-stacked tunneling. However, a few scholars have carried out relevant research and test on the loess composite foundation, the research on the pile composite foundation in the collapsible loess area is even less, and the existing pile-soil interaction mechanism and bearing law in this area are still unclear.

In this study, a series of laboratory model tests of the rigid pile composite foundation in the loess foundation are conducted. Three working modes, including the single pile, single pile composite foundation, and group pile composite foundation, are explored, respectively, thereby analyzing the settlement deformation, distribution of shaft axial force, exertion degree of side friction resistance, and pile-soil stress ratio. Meanwhile, the joint working mechanism of the pile and soil in the composite foundation and its variation law in each bearing stage are also revealed through the aforementioned laboratory model tests. To some extent, the abovementioned outcomes provide a theoretical basis for the spreading of the pile composite foundation in the loess area of China.

\section{Experimental Protocol}

2.1. Materials. The test soil is taken from the excavation section of the foundation pit in Yangling, Shanxi Province, which belongs to $Q_{3}$ loess of the second terrace of the Weihe River. The basic physical property indexes of Yangling loess are shown in Table 1.

In this model test, the dry density of the soil between piles is controlled to be $1.35 \mathrm{~g} / \mathrm{cm}^{3}$, and the target moisture content is $15 \%$. In the process of soil sample preparation, in order to make the water fully and evenly enter the soil, the layered humidifying with sprayed atomization is adopted, and the final moisture content of the prepared soil sample is $15.6 \%$. Meanwhile, a series of compression tests was conducted with the aforementioned prepared soil. The experimental results indicate that the soil belongs to middle compressibility, with a compressibility factor $a=0.18 \mathrm{MPa}^{-1}$ and compression modulus $E_{\mathrm{s}}=12 \mathrm{MPa}$. The cohesion and internal frictional angle were measured, respectively, through the laboratory consolidation undrained triaxial tests, with the results of $c=32.56 \mathrm{kPa}$ and $\varphi=23.04^{\circ}$.

2.2. Scaling Law. The similarity ratio of this model test is selected as $n=20$, and the corresponding ratio for every parameter can be deduced from the dimensional method, which has been described by Zhang et al. [23]. The detailed scaling law is described in Table 2 , in which $L$ and $F$ represent the dimension of size and force. The detailed similarity condition can be expressed as follows:

$$
\begin{aligned}
& C_{\sigma}=C_{\gamma} C_{l}=n, \\
& C_{\delta}=C_{\varepsilon} C_{l}=n, \\
& C_{\sigma}=C_{E}=n, \\
& C_{\varepsilon}=C_{w}=C_{\mu}=1 .
\end{aligned}
$$

2.3. Model Pile. Considering the feasibility and convenience of the laboratory model test, Nylon rod was selected as the model pile. The diameter is equal to $3 \mathrm{~cm}$, which can simulate the pile in construction with the diameter of $60 \mathrm{~cm}$ 
Table 1: Physical parameters of Yangling loess in this model test.

\begin{tabular}{|c|c|c|c|c|c|c|}
\hline \multirow{2}{*}{ Soil } & \multirow{2}{*}{$\begin{array}{c}\text { Specific gravity } \\
\qquad G_{\mathrm{s}}\end{array}$} & \multicolumn{3}{|c|}{ Particle size distribution (\%) } & \multirow{2}{*}{$\begin{array}{c}\text { Maximum dry } \\
\text { density } \\
\rho_{\mathrm{dmax}} \\
\mathrm{g} / \mathrm{cm}^{3}\end{array}$} & \multirow{2}{*}{$\begin{array}{c}\text { Optimum water } \\
\text { content } \\
w_{\mathrm{op}} \\
\%\end{array}$} \\
\hline & & $\begin{array}{c}\text { Sand }(\mathrm{mm}) \\
2-0.075\end{array}$ & $\begin{array}{c}\text { Silt }(\mathrm{mm}) \\
0.075-0.005\end{array}$ & $\begin{array}{c}\text { Clay }(\mathrm{mm}) \\
<0.005\end{array}$ & & \\
\hline Loess & 2.72 & 0.20 & 67.80 & 32.00 & 1.71 & 18.50 \\
\hline
\end{tabular}

Table 2: Scaling law of laboratory model test.

\begin{tabular}{lccc}
\hline Symbol & Physical meaning & Dimension & Value \\
\hline$C_{l}$ & Similarity ratio of geometry & $L$ & 20 \\
$C_{\gamma}$ & Similarity ratio of specific gravity & $F L^{-3}$ & 1 \\
$C_{\sigma}$ & Similarity ratio of stress & $F L^{-2}$ & 20 \\
$C_{E}$ & Similarity ratio of Young's modulus & $F L^{-2}$ & 20 \\
$C_{\varepsilon}$ & Similarity ratio of strain & 1 & 1 \\
$C_{w}$ & Similarity ratio of water content & 1 & 1 \\
$C_{\delta}$ & Similarity ratio of displacement & $L$ & 20 \\
$C_{\mu}$ & Similarity ratio of Poisson's ratio & 1 & 1 \\
\hline
\end{tabular}

through the aforementioned scaling law. The length of the model pile is $60 \mathrm{~cm}$ (equivalent to $1200 \mathrm{~cm}$ in pile foundation construction). The Young's modulus has been determined to $2.1 \mathrm{GPa}$ (equivalent to $42 \mathrm{GPa}$ in pile foundation construction). The aforementioned indexes of Nylon rod can well simulate the rigid pile in construction.

In order to accurately measure the axial force, six pairs of BX120-5AA resistance strain gauges were symmetrically arranged on the surface of the model pile body, whose detailed position is shown in Figure 1. The process of strain gauge sticking should be careful and precise: the surface of model pile was polished firstly, and the vertical and horizontal center lines should be drawn at the measuring points; then, the bottom layer of the strain gauge is bonded with quick drying glue, and the wiring terminal is pasted to protect the strain gauge; finally, the outer layer of the strain gauge is sealed with paraffin and coated with epoxy resin. For the sake of simulating the friction between the pile and soil, fine sand with a particle size of $1-2 \mathrm{~mm}$ was wrapped outside the model pile with strain gauge and wiring terminal, and the treated model pile (as shown in Figure 2) is dried for standby.

\subsection{Experimental Arrangement and Loading}

2.4.1. Model Test Layout. There is no doubt that the boundary effect plays a vital role in the accuracy of the laboratory model test. Hence, a steel drum with size $(100 \mathrm{~cm}$ in diameter and $100 \mathrm{~cm}$ in height) was used in this experiment. Aiming at the pile composite foundation in the loess area, three groups of model tests, the single pile, single pile composite foundation, and group piles composite foundation, were conducted, respectively. The layout of the model test of the group pile composite foundation is shown in Figures 3 and 4 . In this model test, the buried depth of the model pile is $60 \mathrm{~cm}$ (equivalent to $1200 \mathrm{~cm}$ in pile foundation construction), and the diameter is $3 \mathrm{~cm}$ (equivalent to $60 \mathrm{~cm}$ in pile foundation construction). The minimum distance between the edge of the loading plate and the side wall is

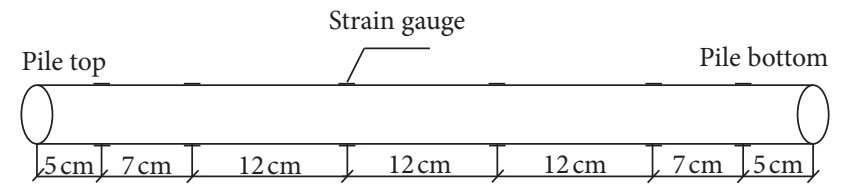

FIGURE 1: Arrangements of resistance strain gages on the model pile.

greater than $10 D$ (in which $D$ is the pile diameter). Meanwhile, the distance between the bottom of the pile and the box is larger than $6 D$, which can efficiently reduce the boundary effect.

2.4.2. Loading Method. The loading method refers to the relevant provisions of GB 50007-2002 and JGJ106-2003, and the slow maintenance load method is adopted. More exactly, the loading is carried out step by step during the test. After the settlement of the previous load is stable, the next load is added until the failure. It should be noted that due to the limitation of loading conditions (the minimum loading unit of the hydraulic jack is $1 \mathrm{kN}$ ), the load of first few stages can only be applied through heaped load, which leads to uneven loading in the early stages. As mentioned above, three groups of laboratory model tests were carried out for the pile composite foundation in the loess area, and the details are shown in Table 3.

\section{Experiment Results and Analysis}

3.1. Analysis of Settlement and Bearing Capacity Characteristics. The $p-s$ (loading-settlement) curve is the macroscopic manifestation of the composite foundation during the working process. In engineering, the ultimate bearing capacity of the foundation is usually determined by the $p-s$ curve. In this model test, the digital displacement sensors are symmetrically arranged on the load plate to reduce the test error, and the overall settlement is measured accordingly. The $p-s$ curve of the single pile, single pile composite foundation, and group piles composite foundation is shown in Figure 5, respectively.

It can be seen that the $p-s$ curves of the single pile and single pile composite foundation are in the form of "elastic $\longrightarrow$ elastic-plastic $\longrightarrow$ plastic." When the upper load is small, the $p-s$ curve is in an elastic stage with very small slope, and the total accumulated settlement is also small. With the increase of vertical load, the $p-s$ curve enters the elastic-plastic stage, the slope of $p-s$ curve increases, and the settlement increases drastically with the rise of load. Compared with the elastoplastic stage in the single pile $p-s$ curve, the single pile composite foundation $p-s$ curve 


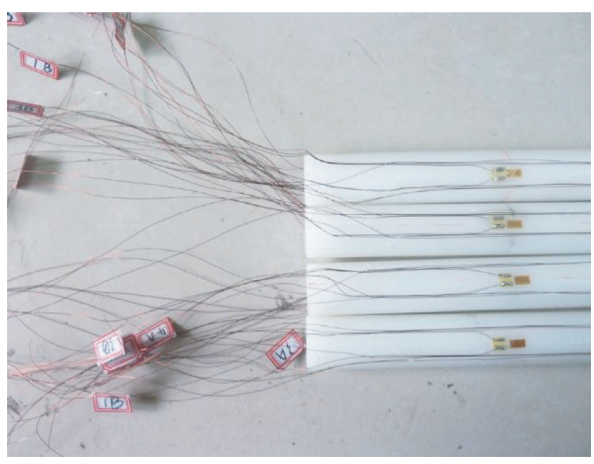

(a)

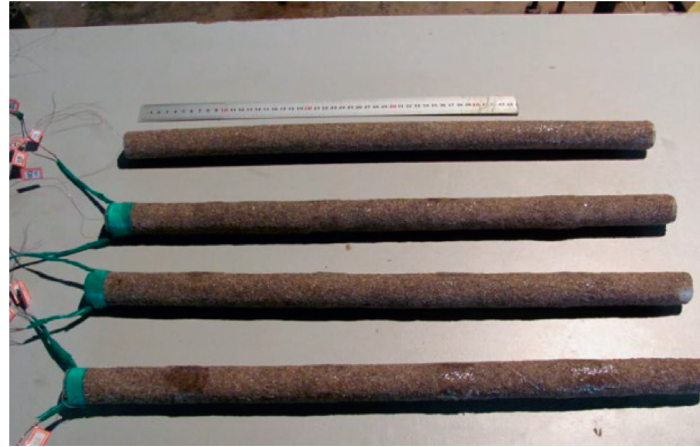

(b)

Figure 2: Treatment of the model pile (strain gauge sticking and sand wrapping).

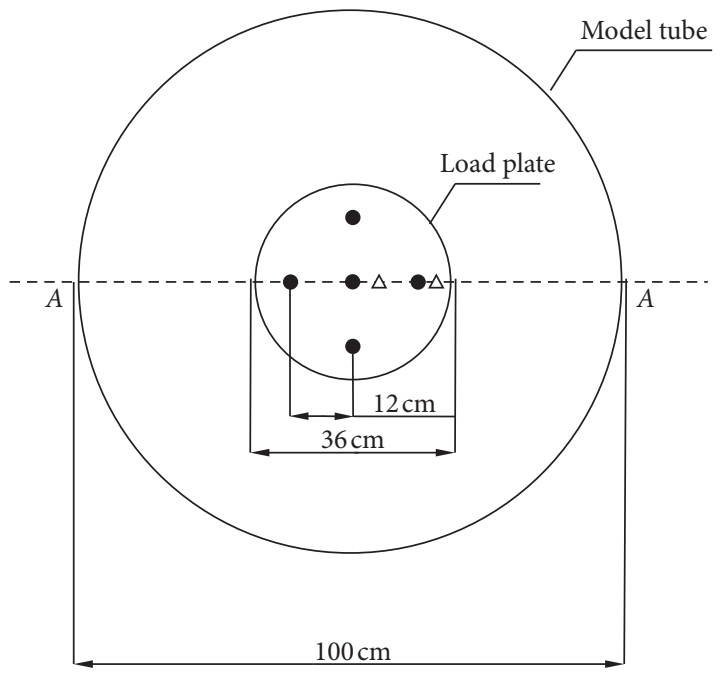

$\Delta$ Earth pressure gauge

- Model pile

FIGURE 3: Laboratory model test arrangement of the group pile composite foundation.

demonstrates a longer range. One possible explanation for this finding could be the bearing characteristics in the composite foundation. In the single pile composite foundation, the pile and soil jointly bear the upper vertical load, and the soil will coordinate the overall settlement deformation. This will bring about a longer elastic-plastic stage in the $p-s$ curve, thereby avoiding the pile sticking into the failure state too early. With further increase of upper load, the settlement of the single pile and single pile composite foundation rises sharply, and the foundation enters into the plastic failure state.

Contrary to the single pile and single pile composite foundation, the $p-s$ curve of the pile group composite foundation shows a gradual failure mode. In detail, the total settlement increases with the increase of upper load, but there is no obvious failure characteristic. The main reason is that when the pile group composite foundation bears the upper vertical load, the interaction between pile and pile (middle pile and side pile) and pile and soil jointly bear the

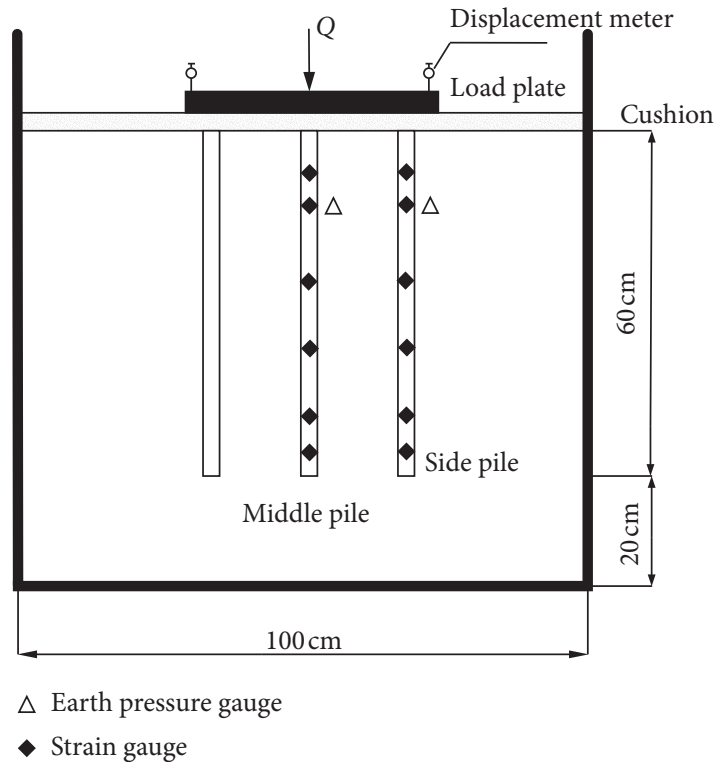

Figure 4: A-A cross section of the group pile composite foundation.

upper load, which ensures that the $p-s$ curve shows no steep drop due to the large overall settlement.

3.2. Distribution Law of Axial Force on the Pile Shaft. The axial force of the pile body reflects the working state of the pile in composite foundation; hence, it is necessary to explore the distribution of axial force along the depth and its characteristics with load variation in the loess foundation. In this model test, the strain value is obtained by measuring the resistance strain gauge; then, the axial force of the pile body at different sections is calculated according to the following formula:

$$
Q_{i}=\bar{\varepsilon}_{i} \cdot E_{i} \cdot A_{i},
$$

where $Q_{i}$ is the axial force at section $i$ of the pile body, $\bar{\varepsilon}_{i}$ is the average strain value on both sides of section $i$ of the pile body, $E_{i}$ is corresponding Young's modulus, and $A_{i}$ is the 
TABLE 3: Experiment protocol for the laboratory model test of the composite foundation.

\begin{tabular}{lccccc}
\hline Material & No. & Experiment items & Pile spacing $(\mathrm{cm})$ & Diameter of the loading plate $(\mathrm{cm})$ & Loading stages \\
\hline \multirow{4}{*}{ Yangling loess } & Y-1 & Single pile & - & - & 6 \\
& Y-2 & Single pile composite foundation & - & 12 & 9 \\
& Y-3 & Group piles composite foundation & 12 & 36 & 12 \\
\hline
\end{tabular}

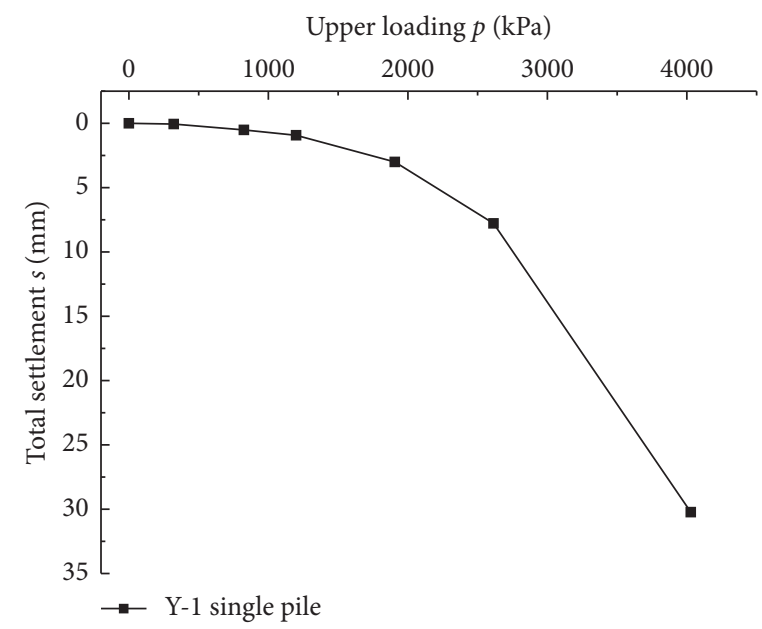

(a)

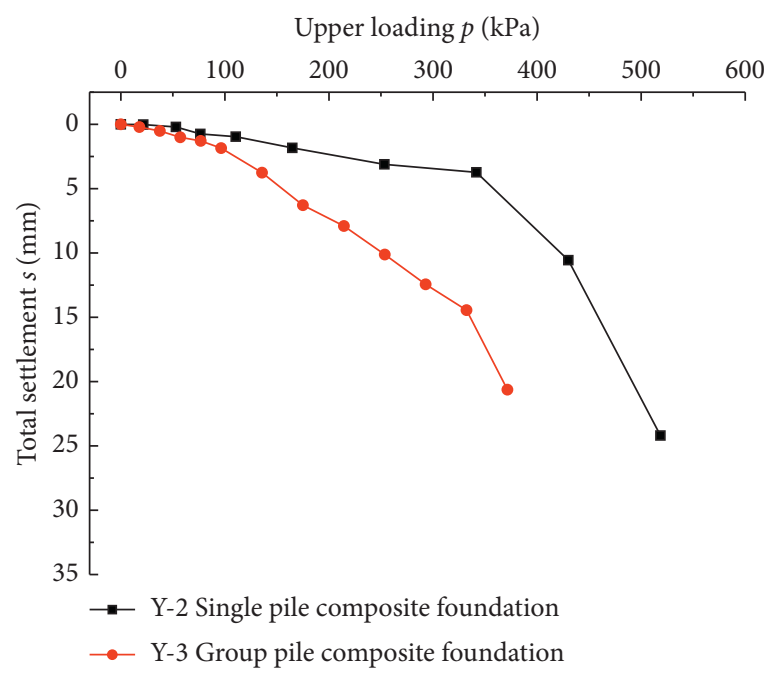

(b)

Figure 5: Loading-settlement curves ( $p-s$ curve) in laboratory model tests. (a) Y-1 single pile; (b) Y-2 single pile, and Y-3 group pile composite foundation.

cross-sectional area of section $i$. Based on the axial force at different sections of the pile body under each loading stage, the distribution diagram of axial force is drawn and shown in Figure 6.

It can be seen that the axial force of the pile body increases with the rise of upper load in three groups of tests. The difference lies in the vertical load test of the single pile, the maximum axial force appears at the top of the pile, and the axial force decreases along the depth, which results from the exertion of the pile side friction. While in the test of the single pile composite foundation and group pile composite foundation, the maximum value of axial force is located in the middle of pile body in the initial stage, rather than the top; however, with an increase of upper load, the location of maximum axial force moves upward until the top of pile, and afterwards, the axial force decreases along with the depth from the top to the bottom of pile. The abovementioned phenomenon can be attributed to negative friction appearing on the pile shaft. More exactly, the negative friction will bring about a downward load, which results in the maximum axial force not appearing in the top. When reaching a critical upper load, the negative friction area disappears and the location of maximum axial force moves to the top. It should be noticed that the aforementioned critical upper load is quite similar for the single pile composite foundation and group pile composite foundation, approximately equal to $330 \mathrm{kPa}$ to $340 \mathrm{kPa}$.
3.3. Distribution Law of Pile Side Friction. Pile side friction is an important part of load transfer in the pile-soil system of the composite foundation: when the upper load acts on the top of the pile, the pile body is compressed and a downward displacement relative to the soil appears, thus forming an upward friction (defined as positive friction); when the settlement deformation of the soil part is greater than that of the pile body, the pile moves upward relative to the soil, resulting in a downward friction (defined as negative friction). The exertion degree of pile side friction directly affects the distribution of pile axial force. However, it is difficult to directly measure the pile side friction, which is usually obtained by a simplified calculation method, and the calculation formula is as follows:

$$
\bar{q}_{i}=\frac{Q_{i}-Q_{i+1}}{u \cdot l_{i}} .
$$

Here, $\bar{q}_{i}$ is the average side friction resistance between the $i$-th section and the $i+1$ section, $Q_{i}$ is the axial force at section $i$ of the pile body, $i$ is the sequence number of the pile detection section, $u$ is the perimeter of the pile shaft; and $l_{i}$ is the pile length between section $i$ and section $i+1$. In order to simplify the analysis, it is assumed that the pile side friction calculated by the abovementioned formula is the friction resistance at the midpoint of two sections (i.e., the middle point of the adjacent resistance strain gauge). Based on the abovementioned assumption, the distribution of pile side 


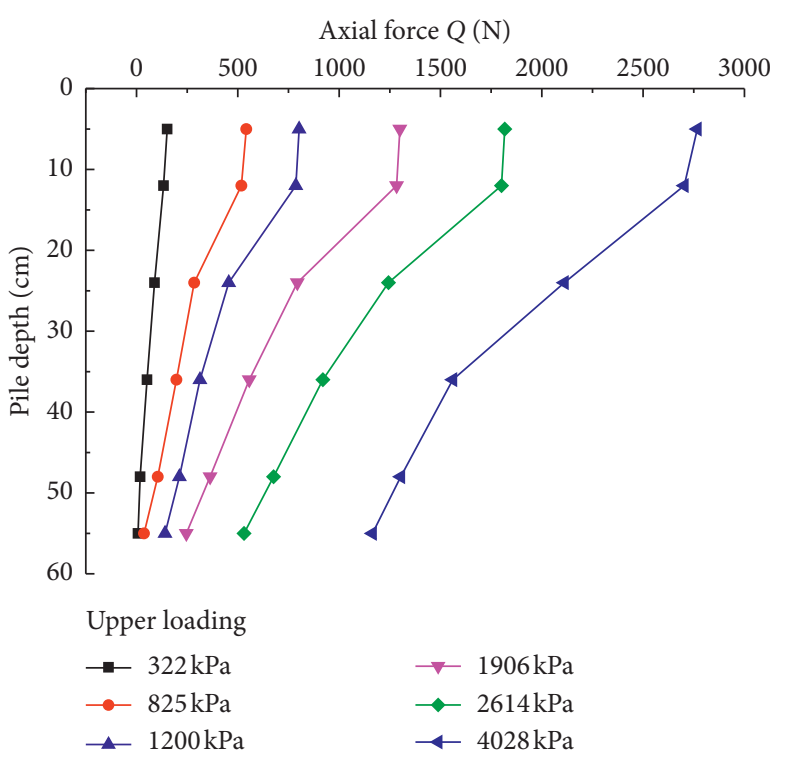

(a)

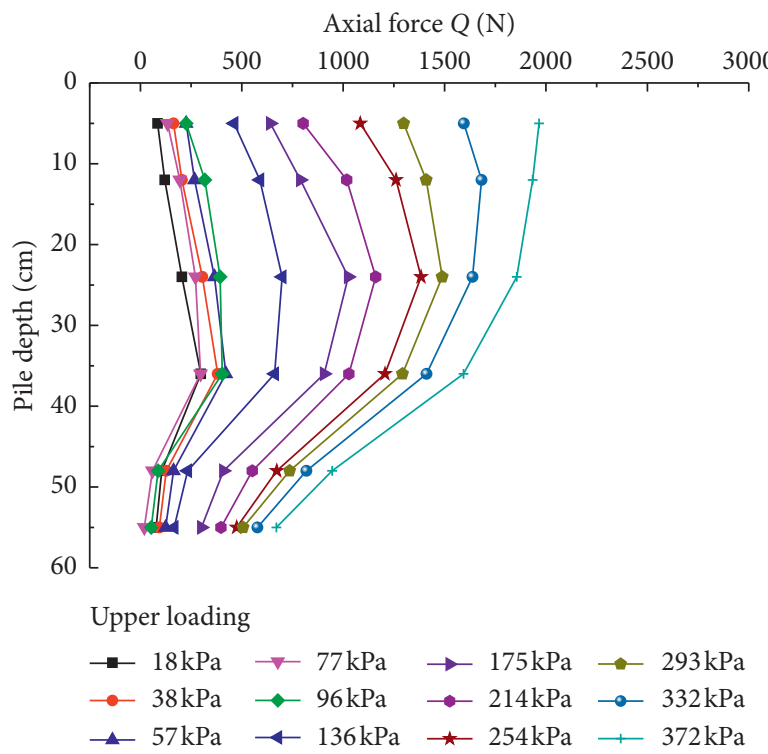

(c)

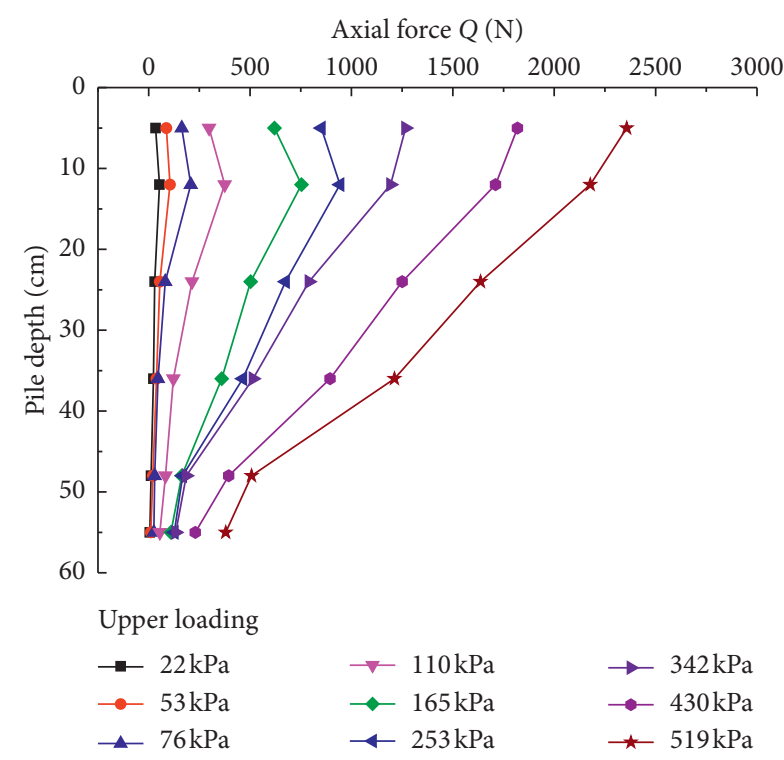

(b)

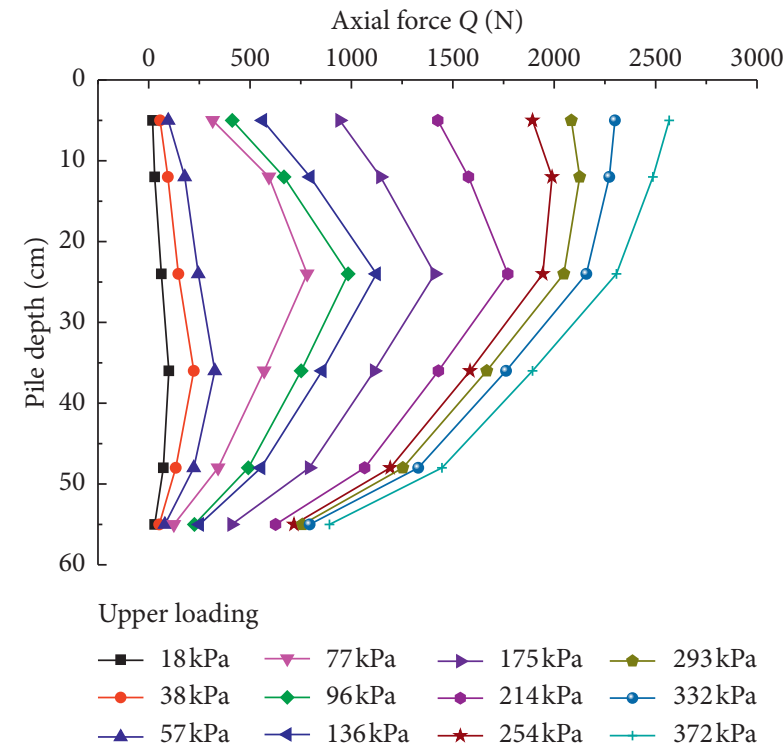

(d)

Figure 6: Variations of pile axis stress with the change of depth and upper load in different model tests. (a) Y-1 single pile, (b) Y-2 single pile composite foundation, (c) Y-3 group pile composite foundation (Middle pile), and (d) Y-3 group pile composite foundation (side pile).

friction resistance under different loads and various tests is illustrated in Figure 7.

It can be seen from Figure 7 (a) that the side friction of the single pile is always positive from the beginning to the end of the upper loading process. Under each stage of load, the friction resistance develops nonlinear with the depth from top to bottom, and the maximum side friction resistance appears at $18 \mathrm{~cm}$ of the pile body. Figures $7(\mathrm{~b}) \sim 7(\mathrm{~d})$ shows that the negative skin friction appears at the upper part of the pile body at the initial stage of loading, both in the single pile composite foundation and group pile composite foundation. This can be explained by the jointed working mechanism between the soil and pile in the composite foundation. When the soil settlement adjacent to the pile is larger than the pile settlement at the same elevation, negative skin friction would occur. At an elevation where the pile and the soil settlements are the same, there would be no load transfer, and this elevation is termed as the neutral plane. Beneath the neutral plane, there would be the usual positive skin friction as the pile settles more than the adjacent soil below this elevation. When increasing to a certain level of load in various tests, the negative friction zone disappears, and the positive skin friction weakens the axial force of the pile body, which is consistent with the distribution law of axial force of the pile body in Section 2.2. In addition, under different levels of load, the maximum positive friction resistance in the single pile composite foundation is located at $42 \mathrm{~cm}$ of the pile body, the maximum positive friction 


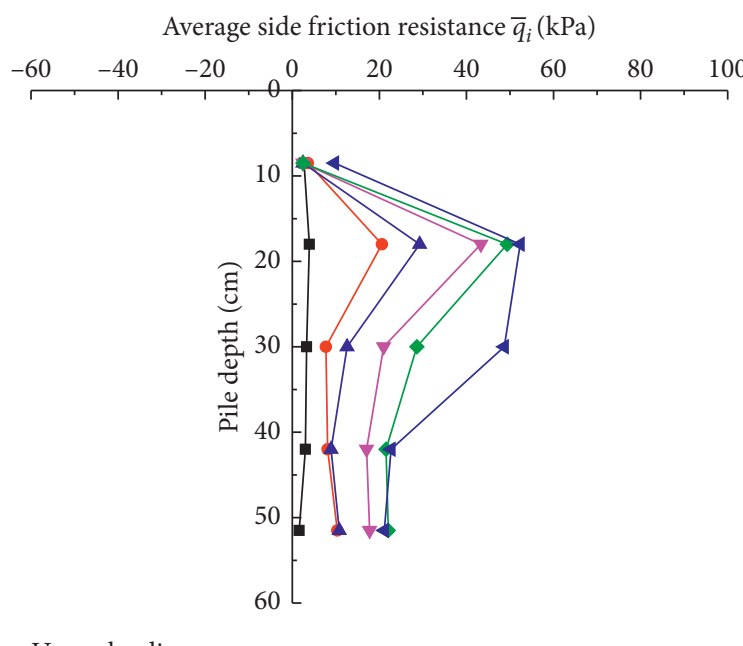

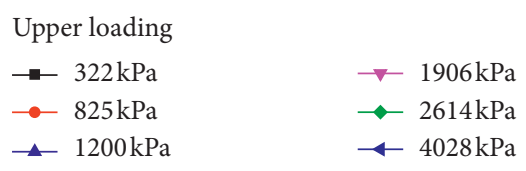

(a)

Average side friction resistance $\bar{q}_{i}(\mathrm{kPa})$

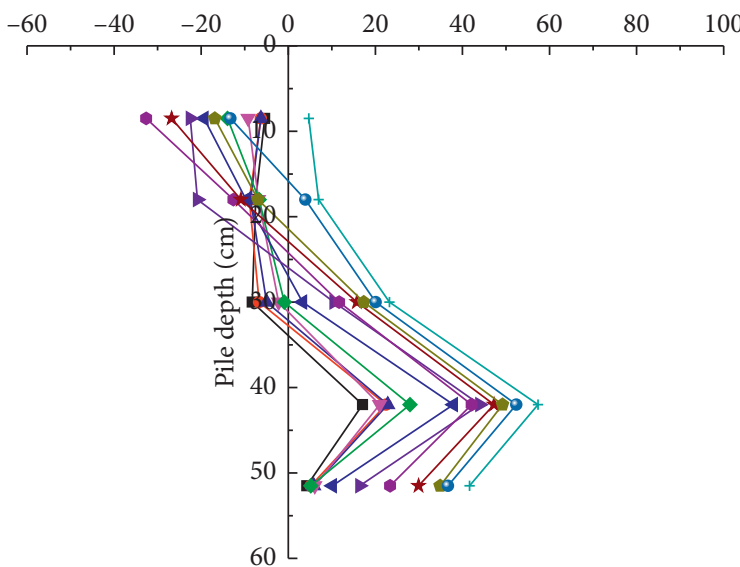

Upper loading

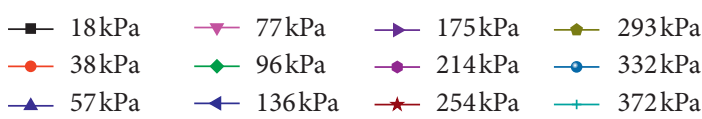

(c)
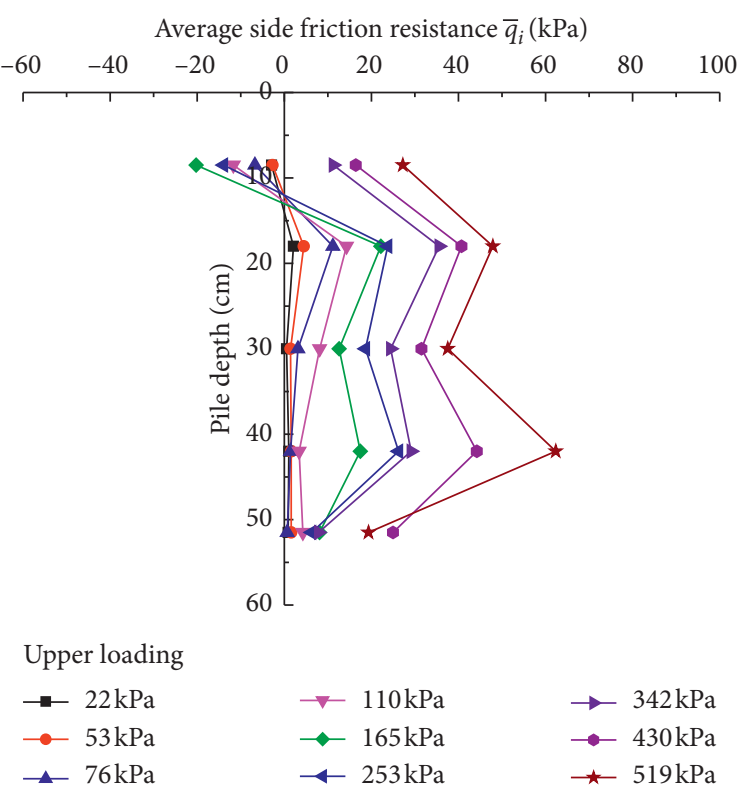

(b) Average side friction resistance $\bar{q}_{i}(\mathrm{kPa})$

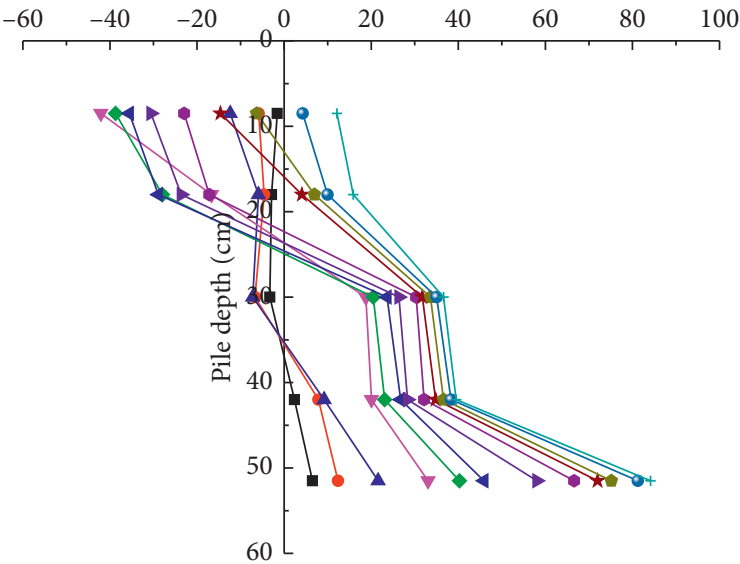

Upper loading

$\rightarrow 18 \mathrm{kPa} \rightarrow 77 \mathrm{kPa} \rightarrow 175 \mathrm{kPa} \rightarrow 293 \mathrm{kPa}$

$\multimap 38 \mathrm{kPa} \longrightarrow 96 \mathrm{kPa} \longrightarrow 214 \mathrm{kPa} \multimap 332 \mathrm{kPa}$

$\neg 57 \mathrm{kPa} \longleftarrow 136 \mathrm{kPa}$ - $254 \mathrm{kPa} \leftarrow 372 \mathrm{kPa}$

(d)

Figure 7: Variations of side friction resistance with change of depth and upper load in the different model test. (a) Y-1 single pile, (b) Y-2 single pile composite foundation, (c) Y-3 group pile composite foundation (middle pile), and (d) Y-3 group pile composite foundation (side pile).

resistance of the middle pile in the group pile composite foundation is located at $42 \mathrm{~cm}$ of the pile body, and the maximum positive friction resistance of the side pile appears at $51.5 \mathrm{~cm}$ of the pile body, all of which are near the pile tip.

In order to analyze the variation trend of pile side friction with load at different depths, the middle pile and side pile in the pile group composite foundation are taken as an example, and the corresponding variation is shown in Figure 8 .

It can be seen that the negative skin friction occurs at $8.5 \mathrm{~cm}, 18 \mathrm{~cm}$, and $30 \mathrm{~cm}$ of the middle pile and side pile, and the following law can be deduced: at the beginning of loading, the relative displacement of pile-soil is small, and the value of negative friction is also small; with the increase of load, the relative displacement of pile-soil increases, and the negative friction resistance gradually develops; the negative skin friction of middle pile and side pile in different depth reaches the peak value under different levels of load. Then, with the increase of load, the negative friction gradually decreases and turns into positive. On the contrary, the friction resistance located in $42 \mathrm{~cm}$ and $51.5 \mathrm{~cm}$ of the pile body is positive from the beginning to the end of 


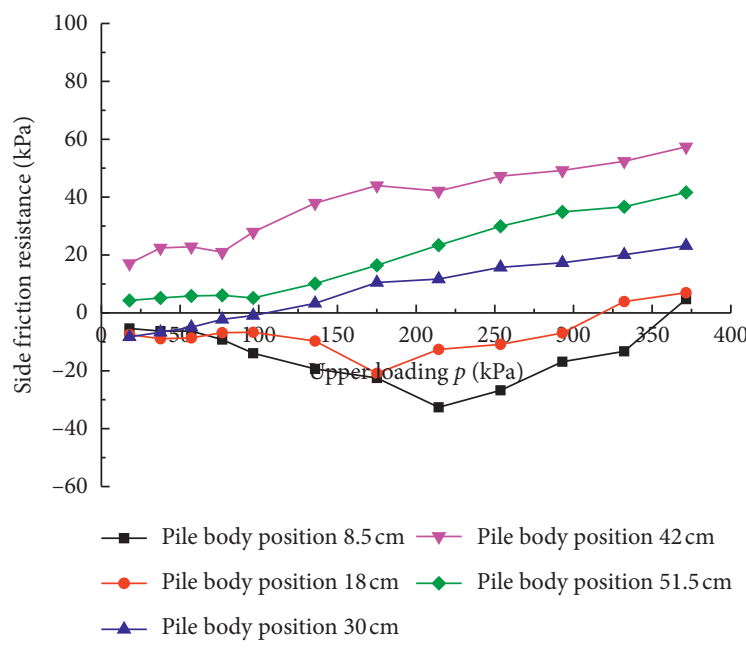

(a)

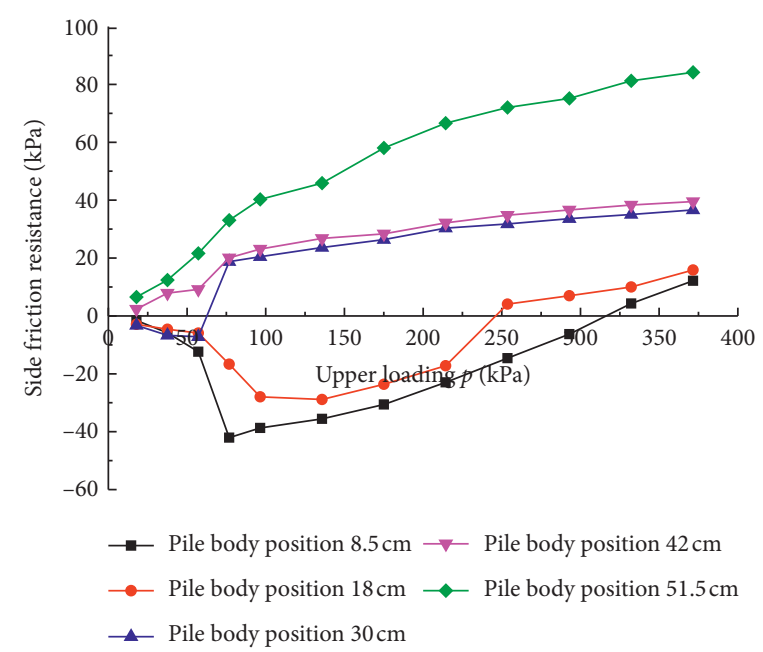

(b)

FiguRE 8: Changes of side friction resistance with the growth of upper load in the group pile composite foundation. (a) Y-3 group pile composite foundation (middle pile); (b) Y-3 group pile composite foundation (side pile).

loading. Meanwhile, the aforementioned positive friction resistance increases continuously with the rise of the upper load.

3.4. Pile-Soil Stress Ratio. The pile-soil stress ratio is an important parameter reflecting the working performance of the pile and soil in the composite foundation, and the distribution of pile stress and soil stress in the composite foundation is uneven. Due to the technical requirements, the earth pressure gauge is embedded at a certain depth (equal to $12 \mathrm{~cm}$ ) in the group pile composite foundation. Hence, the pile stress at $12 \mathrm{~cm}$ is taken for comparison, thereby exploring the corresponding variation law of pile stress ratio.

Figure 9 shows the evolution of pile stress and soil stress in the middle pile and side pile, respectively. It can be seen that, with the increase of the upper load, the stress of the middle pile and side pile increases gradually. However, it should be noticed that the stress of the side pile is obviously greater than that of the middle pile, which is related to the distribution of base pressure under the load plate. In detail, the circular load plate used in the test is equivalent to the rigid foundation acting on the hard clay, and it still keeps as plane after the settlement. A phenomenon of stress concentration appears at the edge of load plate, bringing about a saddle-shaped stress distribution. Therefore, the stress of the side pile is much higher than that of the middle pile. Besides, the measured pile stress is much higher than soil stress in this laboratory composite foundation test. As shown in Figure 10, the pile-soil stress ratio decreases firstly and then increases with the rise of the upper load. The results show that the load-sharing effect of soil is obvious in the loess composite foundation, which leads to the decrease of the pile-soil stress ratio. With the increase of load, the pile bears the main load, and the pile-soil stress ratio increases correspondingly.

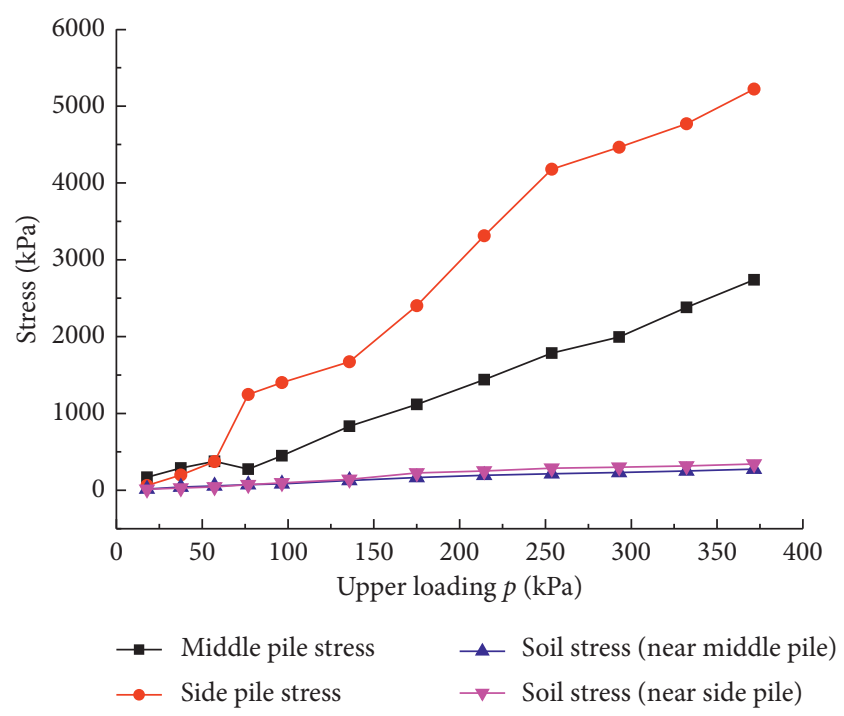

Figure 9: Changes of pile and soil stress with the growth of the upper load in the group pile composite foundation.

\section{Nonlinear Settlement Calculation of the Single Pile Composite Foundation}

Due to the complexity of the constitutive model and pile-soil coupling theory, it is difficult to accurately calculate the settlement of the composite foundation. Combined with the measured loading-settlement curve, the tangent modulus method proposed by Yang is modified and applied in this study [24]. Firstly, the traditional compression modulus is replaced by the tangent modulus that can better reflect the stress condition of the composite foundation, and then, a nonlinear settlement calculation is conducted on the single pile composite foundation, through the layered settlement calculation method. Hyperbolic fitting is selected for the $p-$ 


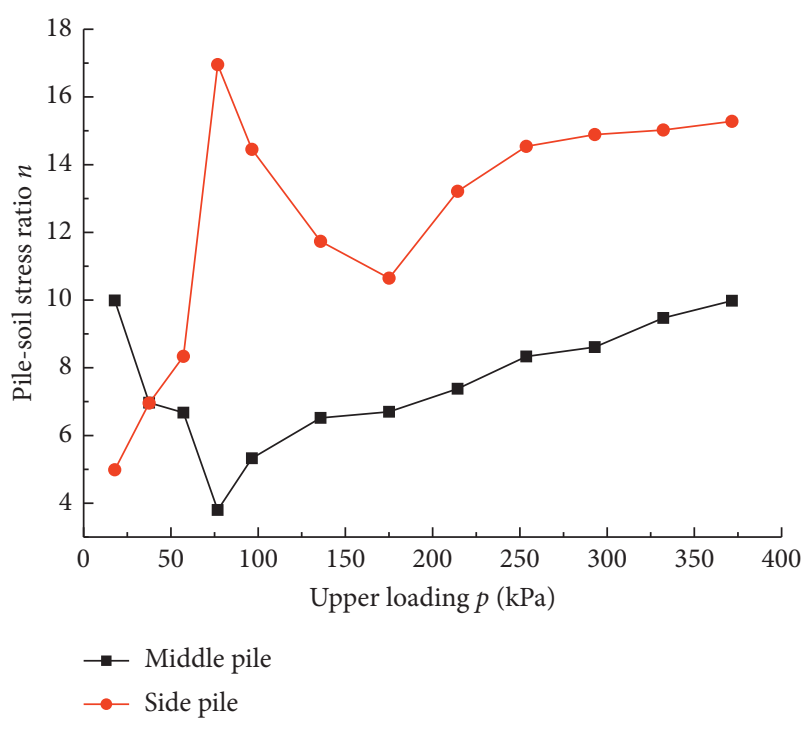

FIGURE 10: Variation of the pile-soil stress ratio with the growth of upper load in the group pile composite foundation.

$s$ curve to obtain a parameter which is indispensable to the decision of the initial tangent modulus, as shown in Figure 11. The hyperbolic fitting function is expressed as follows:

$$
p=\frac{s}{a+b s} \text {. }
$$

The initial tangent modulus of the single pile composite foundation is calculated according to the following formula:

$$
E_{0}=\frac{D\left(1-\mu^{2}\right) \omega}{a} .
$$

In the formula, $D$ is the load plate diameter $(D=12 \mathrm{~cm})$, $\mu$ is Poisson's ratio $(\mu=0.3), \omega$ is the geometric coefficient $(\omega=0.8)$, and $a$ represents the parameter deduced from the abovementioned hyperbolic fitting. The composite foundation is divided into a reinforcement area and underlying layer, whose thicknesses are $60 \mathrm{~cm}$ and $20 \mathrm{~cm}$, respectively. The total depth of the composite foundation is set as 8 layers, with the layer thickness equal to $10 \mathrm{~cm}$. The ultimate bearing capacity $p_{u}$ of the composite foundation in the reinforcement area is calculated according to equation (6). On the contrary, the reinforcement effect of the pile is not considered in the underlying area, and the corresponding $p_{u}$ is the ultimate bearing capacity of soil.

$$
p_{u}=m p_{\text {pile }}+(1-m) p_{\text {soil }} .
$$

Here, $m$ is the replacement rate of the composite foundation, in the model test of the single pile composite foundation, $m=0.0625 ; p_{\text {pile }}$ is the vertical ultimate bearing capacity of the single pile, which is determined directly from the $p-s$ curve of the single pile in Figure $5, p_{\text {pile }}=2615 \mathrm{kPa}$; and the ultimate bearing capacity of $p_{\text {soil }}$ is calculated according to Terzaghi's ultimate bearing capacity formula, meanwhile corrected by the local shear failure mode under a circular foundation:

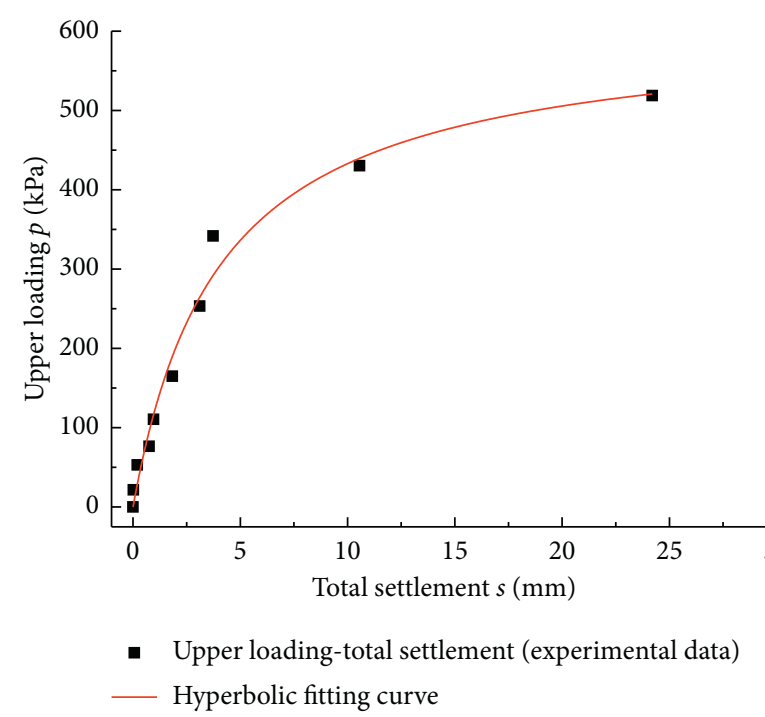

Figure 11: Fitted $p-s$ hyperbolic curve of the single pile composite foundation.

$$
p_{\text {soil }}=0.8 c N_{c}^{\prime}+q N_{q}^{\prime}+0.6 \gamma b N_{r}^{\prime} \text {, }
$$

where $N_{c}^{\prime}, N_{q}^{\prime}$, and $N_{r}^{\prime}$ denote the bearing capacity coefficients, which are related to the internal friction angle $(\varphi)$ of soil, here equal to 13,4 , and 2 , respectively; by conducting the consolidated-undrained test on the soil sample with the same dry density and moisture content as that in the composite foundation test, the cohesion $c=32.56 \mathrm{kPa}$ and $\varphi=23.04^{\circ}, \gamma$ is the unit weight of soil $\left(\gamma=15.6 \mathrm{kN} / \mathrm{m}^{3}\right) ; b$ is the radius of the circular load plate $(b=6 \mathrm{~cm})$; and $q$ is the influence of buried soil on bearing capacity, which is calculated as $q=\gamma h$. Based on the calculation method proposed by Yang, the tangent modulus of the composite foundation at different depths can be determined by the following equation:

$$
E_{t_{i}}=\left(1-\frac{p}{p_{u_{i}}}\right)^{2} E_{0}
$$

where $E_{t_{i}}$ represents the tangent modulus of every layer (counting $i$ from 1 to 8 ), $p$ is the current upper load, $p_{u_{i}}$ is the ultimate bearing capacity in the corresponding composite foundation layer, and $E_{0}$ represents the initial tangent modulus calculated by equation (5). It is assumed that $p$ and $\Delta p$ conform to the distribution of additional stress under circular uniform load, in which $\Delta p$ denotes the increment load. Also, the settlement of every layer can be calculated separately according to the following equation:

$$
\Delta s_{i}=\frac{\Delta p \alpha_{0 i} \Delta h}{E_{t_{i}}}
$$

Here, $\Delta s_{i}$ is the settlement of every layer under the increment load $\Delta p, \alpha_{0 i}$ is the distribution coefficient of additional stress, and $\Delta h_{i}$ denotes the layer thickness (equal to $10 \mathrm{~cm}$ ). The total settlement $\Delta s$ caused by the incremental load $\Delta p$ can be determined as follows: 


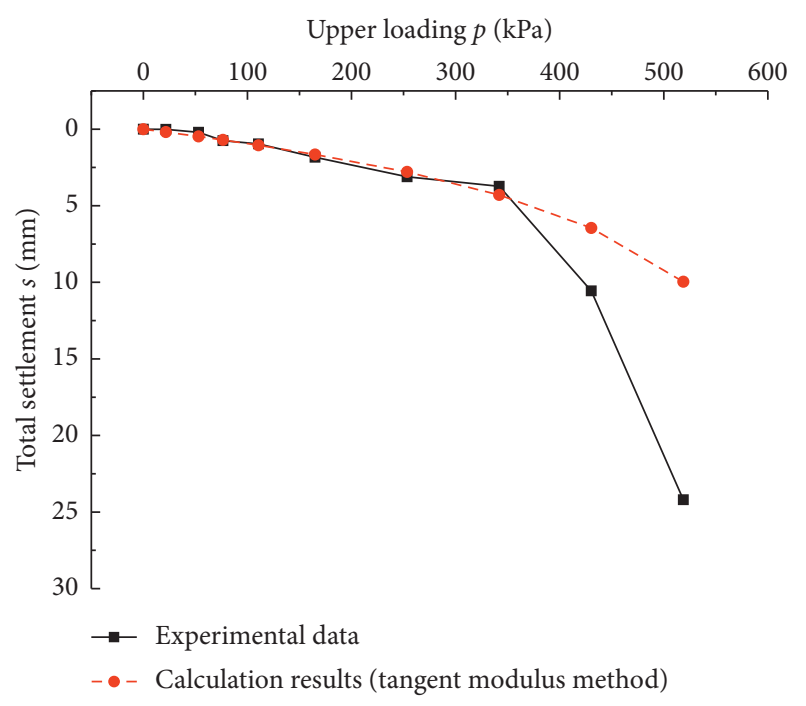

Figure 12: Comparison between calculated settlements and experimental data for the single pile composite foundation.

$$
\Delta s=\sum_{i=1}^{8} \Delta s_{i} .
$$

Figure 12 presents the comparison between calculated settlements and experimental data for the single pile composite foundation. The comparison shows a good agreement between experiments and simulations in the $p-s$ plane. However, it should be noticed some deviations are observed when the upper load is relatively large. Most exactly, the predicted settlement is far less than the experimental data. One possible explanation is that the tangent modulus of the composite foundation is deduced from the hypothesis of elastomer, which cannot well predict the total settlement at the later loading stage. It can be seen the prediction by the aforementioned method shows a good agreement when the $p-s$ curve is located in the elastic and elastic-plastic stage. When the upper load is relatively large, the plasticity failure appears and the hypothesis of elasticity is not applicable any more. At this time, the settlement calculated by the tangent modulus method is quite different from the measured value. It is suggested that the tangent modulus of the plastic failure stage should be modified to make this method more suitable for nonlinear settlement calculation of the composite foundation.

\section{Conclusions}

A series of laboratory tests of the pile composite foundation aiming at the loess are conducted in this study, to explore the working mechanism of the pile and soil in the composite foundation and its variation law in different bearing stages; the tangent modulus method is modified and applied to calculate the total settlement of the single pile composite foundation. A good agreement is generally obtained between the predictions and experimental data until plastic failure appears in the composite foundation. The preliminary conclusions and suggestions are as follows:
(1) The $p-s$ curves of the single pile and single pile composite foundation is obviously in the form of "elasticity to elastoplasticity to plasticity" and accompanied by a sharp drop point. Under the effect of the "group pile effect," the $p-s$ curve of the pile group composite foundation shows a slow variation, and there is no obvious failure point.

(2) The axial force of the pile body increases with the rise of upper load. At the initial stage of loading, the axial force of the pile body in the composite foundation is small at both top and bottom and large in the middle part. When arriving at a certain load, the maximum axial force moves to the pile top, meanwhile decreasing along with the pile depth.

(3) In the single pile foundation test, the side friction is always positive. While in the single pile and group pile composite foundation tests, a neutral point appears in the pile body, bringing about the effect of negative friction above the aforementioned neutral point.

(4) The measured pile-soil stress ratio varies with the rise of the upper load, which obviously illustrates the load-sharing effect of soil in the loess composite foundation. In addition, the pile stress of the side pile is much higher than that of the middle pile due to the saddle-shaped stress distribution at the edge of the load plate.

(5) The modified tangent modulus method can accurately predict the settlement of the composite foundation in a certain load range. A further modification is suggested to be conducted in the future work, thereby extending its application in the nonlinear settlement calculation of the composite foundation.

\section{Data Availability}

The data used to support the findings of this study are available from the corresponding author upon request.

\section{Conflicts of Interest}

The authors declare that they have no conflicts of interest.

\section{Acknowledgments}

This research was financially supported by the fellowship of the National Natural Science Foundation of China (51978572), the fellowship of the China Postdoctoral Science Foundation (2020M673320), the Natural Science Foundation of Shaanxi Province (Nos. 2020JQ-369 and 2020JQ361), and the Fundamental Research Funds for the Central Universities, CHD (Nos. 300102210308, 300102210305, and 300102210307).

\section{References}

[1] Z. Zhang, Pile Foundation Engineering, China Architecture \& Building Press, Beijing, China, 2007. 
[2] J. Zhang, The Application of Composite Foundation on the Collapsible Loess, Ph. D. Thesis, Chang'an University, Xi'an, China, 2001.

[3] J. Wan, H. Tu, and X. Lin, "The application of slag concrete pile on the composite foundation with collapsible loess," West-China Exploration Engineering, vol. 18, no. 6, pp. 64-66, 2006.

[4] C. Liu, The Settlement Calculation of Compaction Pile Composite Foundation in Collapsible Loess Areas, Ph. D. Thesis, Lanzhou University, Lanzhou, China, 2009.

[5] S. Liu and S. Han, "Discussion about the stress ratio and bearing capacity of soil-lime compacted column," Chinese Journal of Geotechnical Engineering, vol. 18, no. 1, pp. 89-94, 1996.

[6] Q. Nie, J. Liang, L. Han et al., "Study of improvement mechanism of compaction-widening piles by tamping in collapsible loess foundation," Rock and Soil Mechanics, vol. 32, no. 6, pp. 1819-1823, 2011.

[7] X. Fan, Z. Yang, and K. Li, "Effects of the lining structure on mechanical and fracturing behaviors of four-arc shaped tunnels in a jointed rock mass under uniaxial compression," Theoretical and Applied Fracture Mechanics, vol. 112, no. 2, Article ID 102887, 2021.

[8] L. Xu, H. Wong, A. Fabbri et al., "A unified compaction curve for raw earth material based on both static and dynamic compaction tests," Materials \& Structures, vol. 54, no. 5, 2021.

[9] R. Feng, Y. Xie, and X. Yang, "Discussion on design method of DJM-pile compound grounds for embankment," Rock and Soil Mechanics, vol. 28, no. 7, pp. 1487-1490, 2007.

[10] M. Yang and S. Liu, "Field tests and finite element modeling of a prestressed concrete pipe pile-composite foundation," KSCE Journal of Civil Engineering, vol. 19, no. 7, pp. 2067-2074, 2015.

[11] M. A. Soomro, S. R. Samo, A. Saand et al., "Numerical parametric study of settlement and load transfer mechanism of pile group due to twin stacked tunnelling with different construction sequences," European Journal of Environmental and Civil Engineering, vol. 2019, Article ID 1695678, 2019.

[12] M. A. Soomro, D. A. Mangnejo, R. Bhanbhro, N. A. Memon, and M. A. Memon, "3D finite element analysis of pile responses to adjacent excavation in soft clay: effects of different excavation depths systems relative to a floating pile," Tunnelling and Underground Space Technology, vol. 86, pp. 138$155,2019$.

[13] M. A. Soomro, N. Mangi, W. Cheng et al., "The effects of multipropped deep excavation-induced ground movements on adjacent high-rise building founded on piled raft in sand," Advances in Civil Engineering, vol. 2020, Article ID 8897507, 2020.

[14] L. Li, H. Chen, J. Li, and D. a. Sun, "An elastoplastic solution to undrained expansion of a cylindrical cavity in SANICLAY under plane stress condition," Computers and Geotechnics, vol. 132, Article ID 103990, 2021.

[15] M. A. Soomro, "3D finite element analysis of effects of twin stacked tunnels at different depths and with different construction sequence on a piled raft," Tunnelling and Underground Space Technology, vol. 109, Article ID 103759, 2021.

[16] C. Wang, B. Wang, X. Wang et al., "Analysis on settlement controlling effect of pile-net composite foundation on collapsible loess by centrifugal model tests," Journal of the China Railway Society, vol. 33, no. 4, pp. 84-92, 2011.

[17] J.-J. Zheng, Y. Liu, Y.-T. Pan, and J. Hu, "Statistical evaluation of the load-settlement response of a multicolumn composite foundation," International Journal of Geomechanics, vol. 18, no. 4, Article ID 4018015, 2018.

[18] Y. Yi, S. Liu, and A. J. Puppala, "Bearing capacity of composite foundation consisting of T-shaped soil-cement column and soft clay," Transportation Geotechnics, vol. 15, pp. 47-56, 2018.

[19] X. Cheng, W. Jing, C. Yin, and C. Li, "Stability parameter analysis of a composite foundation of an oil storage tank in a loess area treated with compaction piles," Soils and Foundations, vol. 58, no. 2, pp. 306-318, 2018.

[20] H. J. Sun and S. Y. Zhang, "A field experimental study of composite foundation with rammed soil-cement pile in collapsible loess area," Advanced Materials Research, vol. 1030-1032, pp. 969-973, 2014.

[21] M. A. Soomro, N. Mangi, H. Xiong et al., "Centrifuge and numerical modelling of stress transfer mechanisms and settlement of pile group due to twin stacked tunnelling with different construction sequences," Computers and Geotechnics, vol. 121, Article ID 103449, 2020.

[22] M. A. Soomro, M. Kumar, H. Xiong, D. A. Mangnejo, and N. Mangi, "Investigation of effects of different construction sequences on settlement and load transfer mechanism of single pile due to twin stacked tunnelling," Tunnelling and Underground Space Technology, vol. 96, Article ID 103171, 2020.

[23] Y. Zhang, Z. Song, X. Weng et al., "Model test on treatment of collapsible foundation of loess metro tunnel," Chinese Journal of Rock Mechanics and Engineering, vol. 39, pp. 1-9, 2020.

[24] G. Yang, "Nonlinear settlement computation of the soil foundation with the undisturbed soil tangent modulus method," Chinese Journal of Geotechnical Engineering, vol. 28, no. 11, pp. 1927-1931, 2007. 